\title{
Assessment of exposure-outcome relationship in cross-sectional study designs: is a time dimension mandatory?
}

\begin{abstract}
When conducting epidemiological studies, different study designs exist depending on the objectives and the study population. Among these designs, one is a cross-sectional study. Despite its several merits, especially among low and middle income countries where the disease burden is heavy, several reports based on cross-sectional study designs suffer in their credibility; one of which is difficulties in controlling of potential bias. In this review, we discuss another irregularity that is most common when analyzing secondary data to assess exposure-outcome association in analytical cross-sectional studies.
\end{abstract}

Keywords: Analytical study, cross-sectional study design, exposure-outcome, secondary data
Volume 9 Issue 4 - 202 I

\author{
Method Kazaura \\ Department of Epidemiology and Biostatistics, Muhimbili \\ University of Health and Allied Sciences, Tanzania
}

Correspondence: Method Kazaura, Department of Epidemiology and Biostatistics, Muhimbili University of Health and Allied Sciences, Dar es Salaam. P. O. Box 65015, Dar es Salaam,Tanzania, Email methodkazaura@gmail.com

Received: September 27, 202 I | Published: October II, 202 I

\section{Introduction}

Cross-sectional study design is among the most common designs in rapid assessments and for large population representative surveys. It is the principal design when estimating the prevalence. This is the reason why, sometimes cross-sectional studies are referred to as prevalent studies. ${ }^{1}$ The advantages and limitations of crosssectional studies have been discussed earlier. ${ }^{2,3}$ Some of the primary limitations and of critical importance are the limited ability to assess causal relationship between the exposure and the outcome of interest and to control potential bias. Inability to assess causality happens, specifically, in analytical studies when estimating the association between the exposures and the outcome. Besides this limitation, another caution should be considered when planning to conduct crosssectional analytical study. In this review, we give some examples by comparing some reports that were based on two sources of data, the primary and secondary data.

\section{Analytical studies using primary and secondary data}

Generally, the main aim of analytical studies is to examine the relationship between the exposure and the outcome. Although Kesmodel $^{4}$ suggests that "there is no time dimension in cross-sectional studies, therefore no time interval between exposure and outcome", a caution should be highlighted because in analytical studies, both the exposure and the outcome must be assessed simultaneously, not only at a single point in time but at the same time. ${ }^{5}$ Deviation to this principle is not very common when using primary data, but it often does when analyzing secondary data. Sources for the major concern are when the exposure changes naturally over the period of time. While examples of individual-level exposures that change naturally include age and income, those of fixed exposures are a particular medical treatment, sex or race. Across-sectional study was conducted in Singapore to assess the association between mushroom consumption and mild cognitive impairment using primary data. ${ }^{6}$ In this study, mushroom consumption was the main exposure of interest. Some of the adjusting variables included age, sex, education, cigarette smoking, etc. The outcome variable was mild cognitive impairment (MCI). Of important to note is that the exposure variable, the pre- determined confounding variables and the main outcome variable were all assessed simultaneously and at the same time. Therefore, when they conclude that "compared with participants who consumed mushrooms less than once per week, participants who consumed mushrooms $>2$ portions per week had reduced odds of having MCI (odds ratio $=0.43,95 \%$ CI $0.23-0.78, p=0.006$ )", methodologically, could be a valid conclusion.

Similarly, in 2019 Mndala \& Kudale ${ }^{7}$ assessed the determinants of overweight and obesity among non-pregnant adult women in Malawi using secondary data, the Demographic and Health Survey of 2015/6.7 The outcome variable was overweight/obesity. Exposure variables were diverse, including age, residence, marital and education status, occupation, religion determinants of overweight/ obesity in Malawi", etc. The input variables for overweight/obesity (weight and height) and all exposure variables were simultaneously assessed during the survey period. Therefore, concluding that, for example, social-economic status and age were highly significant determinants of overweight/obesity among women in Malawi could be a methodological valid conclusion. Comparatively, a 2020-study involving Korean men and women used data set that was collected in 2013. In this study, the outcome variables was use of controlling behaviors. Among the exposures that Ferraresso studied were age, employment status, family income and witnessing violence between parents. ${ }^{8}$ These exposure variables are not fixed. For example age, employment status and family income change with time. While these exposure and confounding variables were assessed in 2013, the outcome variable ("use of controlling behaviors") was reported and recorded in 2013 but could have happened earlier, that is before 2013. Therefore, in principle the outcome variable should have been defined as "use of controlling behaviour in 2013". Ferraresso did not assess the outcome variable and the exposure variables at the same period of time.

A similar setback occurs when using secondary data. For example, various studies have assessed violence and some hypothesized exposures using the Demographic and Health Surveys. In 2020, Izugbara et al..$^{9} \&$ Gubi et al. ${ }^{10}$ \& Lasong et al. ${ }^{11}$ were examining factors associated with either domestic violence or intimate partner violence in some of the sub-Saharan counties. Among the questions 
for the outcomes include "Did your (last) (husband/partner) ever do any of the following things to you (push you, shake you, or throw something at you?)" Another question was "Who has done any of these things to physically hurt you while you were pregnant?" in which one of the options was "Husband/partner". In this survey, one of the questions for assessing the exposures was "What is the current marital status". It is obvious that the outcome and exposures were not assessed simultaneously. Similarly, Yusuf et al. ${ }^{12}$ assessed knowledge and use of modern contraceptives in relation to selected exposures: intimate partner violence, age, residence, education and wealth. In this case, the exposure variables should have been assessed together with the outcome of interest (knowledge and use of contraceptives). Unfortunately, this was not the case. Therefore, although it is not possible to assess the causal relationships between the exposures and the outcomes when using cross-sectional study design, it becomes even more difficult to explain the mechanism of the association when the time period for assessing the exposures differs from that of the outcomes. The main reason for this condition is that if time periods are different, then it will be illogical to compare the outcome differences between the exposed individuals and those unexposed. To conclude, we recommend that, on one hand when planning to conduct a crosssectional analytical study using primary data both the exposure and the outcome variables must be clearly defined prior to data collection. But on the other hand, when analyzing secondary data, a scrutiny of the time period when the exposure and the outcome variables were assessed is mandatory. An extreme mismatch between the time period of assessing the outcomes and exposures would jeopardize the validity of the study and portray the misleading conclusions.

\section{Acknowledgments}

Thanks to Prof Killewo, JZJ and Prof Lie, RT for providing comments and suggestions in the earlier versions of the manuscript.

\section{Conflicts of interest}

The authors declare that they have no conflicts of interest associated with this article.

\section{References}

1. Carneiro AV. Types of clinical studies. III. Cross-sectional studies. Rev Port Cardiol. 2005;24:1281-1286.

2. Setia MS. Methodology Series Module 3: Cross-sectional Studies. Indian J Dermatol. 2016;61:261-264.

3. Muluneh MD, Stulz V, Francis L, et al. Gender based violence against women in sub-Saharan Africa: A systematic review and meta-analysis of cross-sectional studies. Int J Environ Res Public Health. 2020;17:903.

4. Kesmodel US. Cross-sectional studies - what are they good for? Acta Obstet Gynecol Scand. 2018;97:388-393.

5. Pandis N. Cross-sectional studies. Am J Orthod Dentofacial Orthop. 2014;146:127-129

6. Feng L, Cheah IK, Ng MM, et al. The association between mushroom consumption and mild cognitive impairment: a community-based crosssectional study in Singapore. J Alzheimers Dis. 2019;68:197-203.

7. Mndala L, Kudale A. Distribution and social determinants of overweight and obesity: a cross-sectional study of non-pregnant adult women from the Malawi Demographic and Health Survey (2015-2016). Epidemiol Health. 2019;41:e2019039.

8. Ferraresso R. Risk and protective factors associated with intimate partner violence in a nationally representative sample of Korean men. J Prev Med Public Health. 2020;53(2):135-142.

9. Izugbara CO, Obiyan MO, Degfie TT, et al. Correlates of intimate partner violence among urban women in sub-Saharan Africa. PLoS One. 2020;15:e0230508.

10. Gubi D, Nansubuga E, Wandera SO. Correlates of intimate partner violence among married women in Uganda: a cross-sectional survey. BMC Public Health. 2020;20:1008.

11. Lasong J, Zhang Y, Muyayalo KP, et al. Domestic violence among married women of reproductive age in Zimbabwe: a cross sectional study. BMC Public Health. 2020;20:354.

12. Yusuf RA, Dongarwar D, Yusuf ZI, et al. Association between intimate partner violence, knowledge and use of contraception in Africa: comparative analysis across five African regions. Int $J \mathrm{MCH}$ AIDS. 2020;9:42-52. 